Nanoscale Control of $\mathrm{Ag}$ Nanostructures for

Plasmonic Fluorescence Enhancement of Near-Infrared Dyes

Fang Xie ${ }^{1}$, Jing S. Pang ${ }^{1}$, Anthony Centeno ${ }^{2}$,Mary P. Ryan ${ }^{1}$, D. Jason Riley ${ }^{1}$, and Neil M. Alford ${ }^{1}$

${ }^{1}$ Department of Materials and London Centre for Nanotechnology, Imperial College London, London, SW7 2AZ, UK.

${ }^{2}$ Malaysia Japan International Institute of Technology, University Technology Malaysia International Campus, Jalan Semarak, 54100, Kuala Lumpur, Malaysia.

\section{Page Numbers. The font is}

\section{ArialMT 16 (automatically} inserted by the publisher)

\section{Fang Xie, email: f.xie@imperial.ac.uk; www.imperial.ac.uk/people/f.xie}
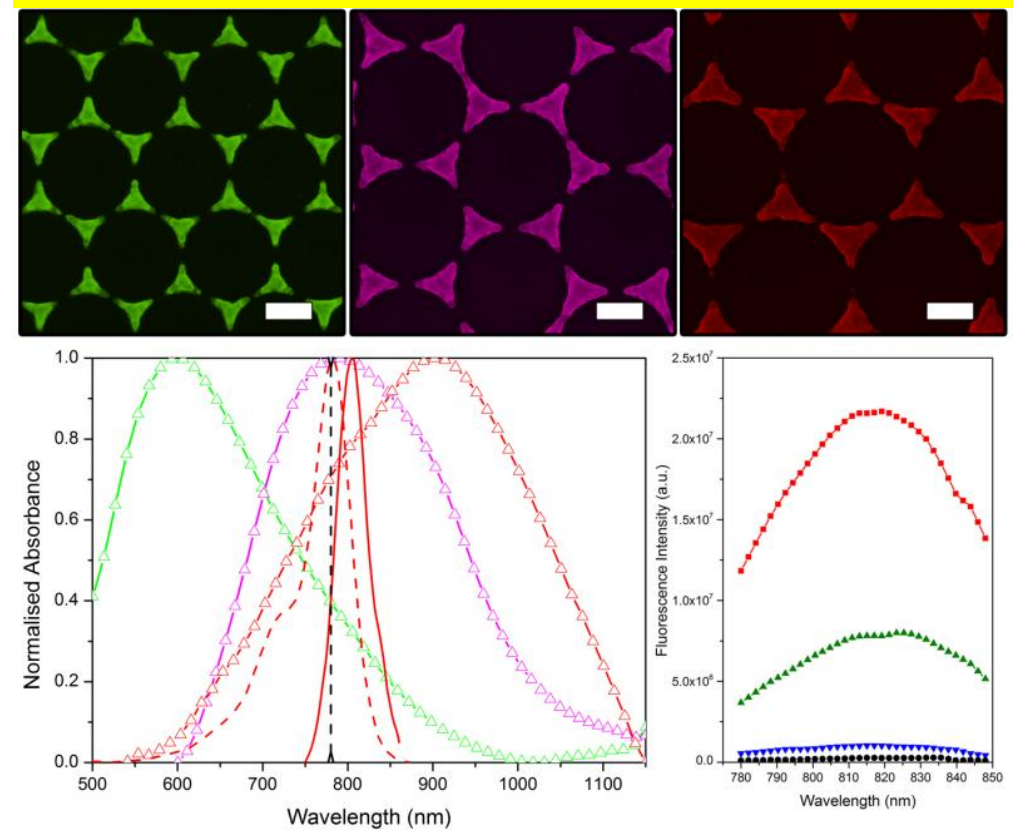

Two orders of fluorescence enhancement were observed for NIR dye by nano-engineering of Ag triangular arrays. Such large area arrays with tunable plasmonic response are excellent candidates for practical sensing applications with high sensitivity and reproducibility. 


\title{
Nanoscale Control of Ag Nanostructures for Plasmonic Fluorescence Enhancement of Near-Infrared Dyes
}

\author{
Fang Xie ${ }^{1}(\bowtie)$, Jing S. Pang, ${ }^{1}$ Anthony Centeno, ${ }^{2}$ Mary P. Ryan, ${ }^{1}$ D. Jason Riley ${ }^{1}$, and Neil M. Alford ${ }^{1}$ \\ ${ }^{1}$ Department of Materials and London Centre for Nanotechnology, Imperial College London, London, SW7 2AZ, UK \\ ${ }^{2}$ Malaysia Japan International Institute of Technology, University Technology Malaysia International Campus, Jalan Semarak, 54100, \\ Kuala Lumpur, Malaysia \\ Received: day month year / Revised: day month year / Accepted: day month year (automatically inserted by the publisher) \\ (C) Tsinghua University Press and Springer-Verlag Berlin Heidelberg 2011
}

\begin{abstract}
Potential utilization of proteins for early detection and diagnosis of various diseases has drawn considerable interest in the development of protein-based detection techniques. Metal induced fluorescence enhancement offers the possibility of increasing the sensitivity of protein detection in clinical applications. We report the use of tuneable plasmonic silver nanostructures for the fluorescence enhancement of a NIR dye (Alexa Fluor 790). Extensive fluorescence enhancement of $\sim 2$ orders of magnitude fold is obtained by the nanoscale control of the $\mathrm{Ag}$ nanostructure dimensions and interparticle distance. These $\mathrm{Ag}$ nanostructures also enhanced fluorescence from dye with very high quantum yield (7.8 fold for Alexa Fluor 488, $Q y=0.92)$. A combination of greatly enhanced excitation and an increased radiative decay rate, leading to an associated enhancement of the quantum efficiency leads to the large enhancement. These results show the potential of Ag nanostructures as MIFE substrate for dyes in the NIR 'biological window' as well as the visible. Ag nanostructured arrays fabricated by colloidal lithography thus show great potential for NIR dyes based biosensing applications.
\end{abstract}

\section{KEYWORDS}

Ag triangular-like nanostructures, fluorescence enhancement, NIR dyes, scattering, absorption.

\section{Introduction}

Improving the capability of fluorescence measurements is recognized as being important for future advances in biology and medicine [1]. The use of fluorescent molecules is currently the most common labelling technique in biosensing and bioimaging for the detection of disease biomarkers and such approaches have seen widespread use in clinical practice due to their versatility, potential for multiplexing, ease of use, and remarkable sensitivity [2].

Fluorescent molecules emitting at wavelengths in the physiologically relevant "water window" (700 - $900 \mathrm{~nm})$, in which penetration depth is high and autofluorescence is minimal, are of particular interest and are potentially an attractive technology for bioapplications [3]. However, the low quantum 
yield and poor photostability of NIR dyes currently limits their applicability. Indeed, the sensitivity of many clinical assays is limited by sample autofluorescence. To design and synthesise Near Infra-red (NIR) dyes with high quantum yield and photostability has proven to be extremely challenging, due to the complex synthetic routes required for these large, complex molecules [4]. The amplification of light from NIR fluorophores by coupling to metal nanostructures is a promising strategy for dramatically improving both the detection sensitivity and image enhancement, thereby realizing the potential advantages of the NIR fluorophores.

Metal induced fluorescence enhancement (MIFE) is now a well-recognized technology wherein the near-field interaction of fluorophores with metallic nanostructures can, under optimized conditions, lead to a substantial fluorescence enhancement [5][7]. The phenomenon of MIFE is attributed to two contributions. Firstly, local enhancements of the electric field take place, induced by the Localized Surface Plasmon Resonances (LSPR) of the metal nanostructures. Secondly, metal nanostructures are able to modify the radiative and non-radiative decay rates of nearby fluorophores, changing both the fluorescence lifetime and quantum yield. Additionally, these nanostructures can affect photostability, and increase energy transfer [8]. Since the discovery of the Purcell effect [9], efforts to increase the sensitivity of fluorescence detection have focused on controlling the local electromagnetic (EM) environment of the fluorophores and taking advantage of the interaction between an emitter and its surroundings. The dielectric environment has a profound influence on the emission of a fluorophore, through its spontaneous emission rate and local modifications of the electromagnetic field: fluorescence quenching [10][12], fluorescence enhancement [13]-[15] or both [16][17] have all been reported. It has been shown experimentally, and supported by theoretical calculations, that the fluorescence enhancement factors of metal nanostructures depend on the particle size, shape, interparticle separation, surrounding dielectric medium, as well as the particle arrangement geometry and distance between the metal and fluorophore. Moreover, metal induced fluorescence critically depends on the spectral overlap between the LSPR in metal nanostructures with the spectral properties of the fluorophore.

Much of the recent work on MIFE for NIR dyes fluorescence enhancement are based on gold (Au) nanostructures including $\mathrm{Au}$ nanorods, $\mathrm{Au}$ nanoshells, porous Au films by dealloying [18], [19], where systematic LSPR tuning is not possible. On the other hand, ordered arrays of $\mathrm{Au}$ based nanoparticles for NIR dyes study, fabricated by electron-beam lithography or related techniques are reproducible and tunable but have an associated high cost and low throughput [20][21]. It has previously been reported that silver $(\mathrm{Ag})$ is a better material for fluorescence enhancement [22]. This is considered to be partly due to the fact that for a LSPR in the visual part of the spectrum Ag nanoparticles will be larger than the equivalent $\mathrm{Au}$ particles. The excitation and emission enhancements that lead to MIFE are thought to be related to the scattering efficiency of a metal nanoparticle. It is well known that for the same incident wavelength Ag particles will have a larger scattering efficiency than $\mathrm{Au}$ nanoparticles [23]. Geddes et al. [24] first reported fluorescence enhancement on indocyanine green using Ag colloids and Anderson et al. reported fluorescence enhancement on IRDye ${ }^{\circledR} 700$, and IRDye ${ }^{\circledR} 800$ using silver island films $[25,26]$. However, these previously reported $\mathrm{Ag}$ based substrates for NIR dye enhancement did not allow for controlled plasmonic. We are not aware of any previous reports on the fluorescence enhancement using silver metal regular arrays with plasmonic tunability for fluorescence enhancement of NIR dyes. In this paper we report the use of silver based nanostructures formed by colloidal lithography for NIR fluorescence enhancement. Colloidal lithography has its distinct advantages including large area, low cost, multiscale, and flexible tuning parameters. By combining colloidal lithography and subsequent oxygen plasma etching, we further adjust the final nanostructures by controlling the size and interparticle distance. Such flexibility makes it a very versatile method for the preparation 
of desired nanostructures with controllable small gaps and a homogeneous MIFE substrate covering a large area $\left(\mathrm{cm}^{2}\right)$.

Ag arrays were fabricated using a polystyrene sphere (PS) template in diameter of $300 \mathrm{~nm}, 500 \mathrm{~nm}$, and $620 \mathrm{~nm}$, respectively. In a second set of samples the polystyrene template were treated with 15 seconds of oxygen plasma etching prior to metal deposition to reduce the separation distance between the particles. For the unetched samples, three Alexa Fluor dyes (Alexa Fluor ${ }^{\circledR}$ 488, Alexa Fluor $^{\circledR}$ 680, Alexa Fluor ${ }^{\circledR} 750$ ) were chosen so that their absorption and emission spectra map onto the measured LSPR spectra of the Ag nanoparticles. For the etched samples Alexa Fluor ${ }^{\circledR} 790$ was chosen to specifically study the fluorescence enhancement effect for NIR dye. Fluorescence enhancement was observed (as shown in Fig. 1 in every case, with a maximum fluorescence enhancement of $\sim 83$ fold from the PS 500 template with 15 seconds oxygen plasma etching. To obtain a better understanding of the role played by the metal nanoparticles, the optical properties of the Ag arrays were investigated along with computational electromagnetic modelling and a lifetime study. Electromagnetic modelling is an indispensable tool to investigate regular nanostructure arrays as the optical properties of such plasmonic structures are dependent on many parameters including dimensions, materials, and surrounding medium. The fluorescent enhancement is discussed in terms of the theoretical framework of MIFE.
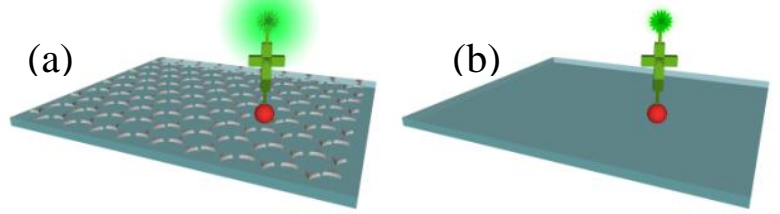

Figure 1 Schematic illustration of fluorescence enhancement on Ag metallic surface and glass surface as a control: (a) Alexa Fluor protein conjugate on Ag triangular-like nanostructures array surface (hcp arrangement), (b) Alexa Fluor protein conjugate on glass surface.

\section{Experimental Section}

\subsection{Materials}

Polystyrene microspheres with diameters of $300 \mathrm{~nm}$, $500 \mathrm{~nm}$ and $620 \mathrm{~nm}$ (10 wt.\%) were purchased from Bangs Laboratories Inc., USA. Streptavidin (SA) conjugated dyes, Alexa Fluor ${ }^{\circledR} 488$ (AF488-SA), Alexa Fluor $^{\circledR} 680$ (AF680-SA), Alexa Fluor ${ }^{\circledR} 750$ (AF750-SA), and Alexa Fluor ${ }^{\circledR} 790$ (AF790-SA) were purchased from Invitrogen. A FluoroProfile Protein Quantification Kit, Phosphate buffer saline (PBS) pH7.4, and biotinlated bovine serum albumin (BSA-Bt) were obtained from Sigma-Aldrich. P-Type silicon wafers, boron-doped (with resistivity of $1-5 \Omega \mathrm{cm}$ ), were purchased from MMRC Inc. Glass microscope slides were purchased from VWR. Nanopure water (> $18.2 \mathrm{M} \Omega$ ), purified using the Millipore Mili-Q gradient system, was used in all experiments.

\subsection{Methods}

\subsubsection{Preparation of Periodic Ag Nanostructure Array}

Polystyrene monolayer templates were prepared by using a modified colloidal lithography method described in detail in reference [17]. Briefly, monodisperse polystyrene (PS) particles with a diameter of $300 \mathrm{~nm}, 500 \mathrm{~nm}$, and $620 \mathrm{~nm}$ were diluted by mixing with an equal amount of ethanol, respectively. Glass substrates (10 by $10 \mathrm{~mm}$ ) were cleaned by immersion in piranha solution (3:1 concentrated $\mathrm{H}_{2} \mathrm{SO}_{4}: 30 \% \mathrm{H}_{2} \mathrm{O}_{2}$ ) at $80{ }^{\circ} \mathrm{C}$ for 1 hour. After cooling, the substrates were rinsed repeatedly with DI water and then sonicated for $60 \mathrm{~min}$ in 5:1:1 $\mathrm{H}_{2} \mathrm{O}: \mathrm{NH}_{4} \mathrm{OH}: 30 \% \mathrm{H}_{2} \mathrm{O}_{2}$ solution. Following sonication they were again rinsed thoroughly with water and used immediately. Approximately 3 to 5 $\mu \mathrm{l}$ of the prepared PS solutions was applied onto the surface of a clean and large ( 30 by $20 \mathrm{~mm})$ silicon wafer, which had been kept in $10 \%$ dodecylsodiumsulfate solution for $24 \mathrm{~h}$ previously. The wafer was then slowly immersed in a $15 \mathrm{~cm}$ glass vessel filled with $150 \mathrm{ml}$ of Milli-Q water; the PS particles form a disordered monolayer on the water surface. To consolidate the particles, the water surface tension was changed by the addition of $4 \mu \mathrm{l}$ of $2 \%$ dodecylsodiumsulfate solution and a large monolayer with highly ordered areas was obtained. Such monolayers were then lifted off from the water 
surface using the glass squares or silicon substrates for SEM.

Once the 2D colloidal crystal template was formed, the substrates, with or without $\mathrm{O}_{2}$ plasma etching, were mounted into the chamber of Mantis e-beam evaporation system, equipped with deposition monitor quartz crystal microbalance, for $\mathrm{Ag}$ deposition with a fixed thickness of $75 \mathrm{~nm}$. The nanosphere mask was removed by sonicating the entire substrate in either $\mathrm{CH}_{2} \mathrm{Cl}_{2}$ or absolute ethanol for $2 \mathrm{~min}$. An array of triangularly shaped particles remains on the substrate. In this study, 2 sets of samples were prepared: first set was Ag arrays from above mentioned PS template with a diameter of $300 \mathrm{~nm}, 500 \mathrm{~nm}$, and $620 \mathrm{~nm}$, respectively (referred to as PS300-0s, PS500-0s, PS620-0s); the second set were Ag arrays from those templates which were treated with 15 second $\mathrm{O}_{2}$ plasma etching prior to metal evaporation, inducing larger triangular structures with smaller gaps (PS300-15s, PS500-15s, PS620-15s).

\subsubsection{Immobilization of Fluorophore-Protein Conjugation Monolayer}

Both the Ag nanoparticle array surface and the glass surface as a control were covered with a self-adhesive silicone/rubber well with a thickness of $2 \mathrm{~mm}$ and diameter of $5 \mathrm{~mm}$. The size of the wells corresponded to the well size routinely used in 96-well plates for high-throughput screening. First, the biotinylated-BSA (bBSA) solution of 100 $\mathrm{mg} / \mathrm{mL}$ in sodium phosphate buffer (50 mM, pH7.2) was added into the wells and incubated for $1 \mathrm{~h}$, and rinsed with water to remove unbounded proteins. This step facilitated the formation of a monolayer of bBSA on both Ag array surface and glass surface. Binding of the streptavidin-Alexa Fluor dye conjugations (AF-SA) was carried out by adding 25 $\mu \mathrm{g} / \mathrm{mL}$ into the wells and incubating for 2 hours. The wells were washed with PBS buffer to remove unbounded streptavidin-dye conjugations.

The formed streptavidin-Alexa Fluor dye monolayer makes it possible to quantitatively compare the fluorescence intensity of fluorophore-protein conjugates with and without various Ag nanostructures on glass (after background signal subtraction and correction for differences in surface coverage). For each Alexa Fluor dye studied here, each sample including the control glass slide was positioned in 2 wells. One was incubated with bBSA only, to establish the fluorescence background used as reference. The other was incubated with streptavidin-Alexa Fluor dyes to form a dye-protein monolayer. The selected Alexa Fluor dyes and their quantum efficiency (Qy) are AF488 with Qy of 92\%, AF680 with Qy of 36\%, AF750 with Qy of $12 \%$, and AF790 with Qy of 4\%, respectively. The fluorescence enhancement factor from the $\mathrm{Ag}$ nanostructures is

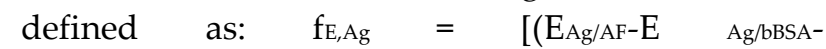

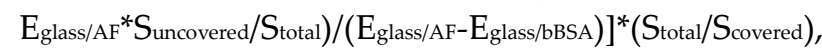
where $\mathrm{E}_{\mathrm{Ag} / \mathrm{AF}}$ is the fluorescence intensity of Alexa Fluor on Ag nanostructure surfaces, $E_{A g / b B S A}$ is the background fluorescence of bBSA on Ag nanostructure surfaces, Eglass/AF is the fluorescence intensity of Alexa Fluor on glass surface, E glass/bBSA is the background fluorescence of bBSA on glass surface, Suncovered is the surface area of uncovered bare glass given a certain masking geometry, and Scovered is the surface area of the Ag triangles, and

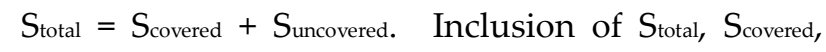
and $S_{\text {uncovered allows one to adjust for the differences }}$ in the total amount of bBSA deposited on various surfaces, as bBSA forms a monolayer on both glass and Ag surface. However, in order to understand the area average of enhancement, which is the useful parameter for applications such as biosensing, we modified the equation to reflect the averaged fluorescence enhancement from Ag samples as a whole rather than the enhancement only from Ag triangular structures. The enhancement factor is therefore defined as: $\mathrm{fE}_{\mathrm{E}}=$ $\left[\left(E_{\text {Ag/AF-EAg/bBSA }}\right) /\left(E_{\text {glass/AF-Eglass/bBSA })}\right]^{*}\left(\right.\right.$ Suncovered $\left._{\text {Stotal }}\right)$. The enhancement factors reported in this paper have used this second method.

\subsection{Characterization}

Scanning electron microscopy (SEM) images of Ag nanostructures after template removal were collected using a LEO Gemini 1525 field emission gun scanning electron microscope (FEG-SEM). The optical properties of the samples from $300 \mathrm{~nm}$ to $1500 \mathrm{~nm}$ were measured at room temperature 
using a Cary 500 spectrometer. All the spectra presented here were obtained using unpolarized light. The fluorescence emission spectra were taken by a Fluorology Tau 3 system from Jobin-Yvon-Horiba with $450 \mathrm{~W}$ Xe lamp excitation. All spectra were corrected for the spectral response, and long-pass filters were used to eliminate the contribution from the scattered excitation light. The samples with AF488-SA were excited at $480 \mathrm{~nm}$, and their fluorescence were measured in the range of $500-650 \mathrm{~nm}$ using $5 \mathrm{~nm}$ slits by spatially averaging the fluorescence from sample in regions of $\sim 1 \mathrm{~mm}$ by $3 \mathrm{~mm}$. The samples with AF680-SA, AF750-SA, and AF790-SA were excited at $670 \mathrm{~nm}$, $740 \mathrm{~nm}$, and $780 \mathrm{~nm}$, respectively. Their fluorescence was measured in the range $690-750 \mathrm{~nm}$, $760-850 \mathrm{~nm}$, and 800-860 nm, respectively. All the other settings were kept the same as sample AF488-SA. Fluorescence decay curves of AF790-SA on metallic surface and glass surface were measured using the time-correlated single photon counting (TCSPC) technique [16] with a FluoTime200 spectrometer (PicoQuant) equipped with a TimeHarp300 TCSPC board (PicoQuant) and a Hamamatsu photomultiplier (PMA-185). The excitation source was a $730 \mathrm{~nm}$ picosecond pulsed diode laser (PicoQuant, LDH730) driven by a PDL800-D driver (PicoQuant) operated at $40 \mathrm{MHz}$ pulse repetition rate (10-). The emission was collected at right angles to the excitation laser beam. The emission arm was fitted with a long pass filter (HQ460LP, Chroma) before the monochromator (Scientech 9030). The full width half maximum (fwhm) of the system's instrument response function (IRF) was 350 ps. The fluorescence decay curves were analysed using the FluoFit software (PicoQuant, version 4.2.1) based on a multi-exponential model which involves an iterative reconvolution process. The quality of the fits was assessed by the value of the reduced $\chi 2$ value and a visual inspection of the distribution of the weighted residuals and their autocorrelation function [27].

\subsection{Computational Electromagnetic Modelling}

The calculations of the optical and electrodynamic properties of Ag arrays were carried out using the finite difference time domain (FDTD) technique [28]. In brief, a 3-D total-field scheme is used with a grid resolution of 1-2 $\mathrm{nm}$ in each direction. The grid resolution for each case was obtained by convergence testing. The dielectric function of the Ag nanoparticles was modelled with a combined Drude-Lorentz model [29] (data provided in supplementary information). To prevent non-physical reflections from the extremities of the FDTD workspace Perfectly Matched Layers (PML) were placed at the boundaries. The transmission and reflection coefficients are calculated by considering the power flow through computational surfaces above and below the nanoparticle array, as previously described in reference [30]. All FDTD calculations were carried out using the MEEP FDTD code [31] on a HPZ800 workstation with two Quad core processors and 64 GBytes of RAM.

\section{Results and Discussion}

\subsection{Ag Nanostructures and Their Plasmonic Resonance}

It is well-known that local field enhancement is highly dependent on the gap size between the nanoparticles. Such an interparticle interaction is demonstrated by the fact that the relative plasmon wavelength shift $(\Delta \lambda / \lambda)$ for polarization along the interparticle axis decays nearly exponentially with the gap distance. In fact, it has been reported both theoretically [32] and experimentally [33] that an asymptotic dependence of the position as a function of spacing parameter exists.

A monolayer of polystyrene spheres (PS) with $300 \mathrm{~nm}, 500 \mathrm{~nm}$, and $620 \mathrm{~nm}$ diameter were deposited directly on a glass surface) by using a self-assembly technique on a water surface, a modified method to form a large area of highly ordered hexagonal closed pack array (hcp) monolayer. Such well-organized monolayers of PS enable us to obtain homogeneous Ag nanotriangular-like arrays after subsequent Ag evaporation and template removal. Fig. 2 shows representative top view SEM images of Ag nanoparticles fabricated in this study, which have 

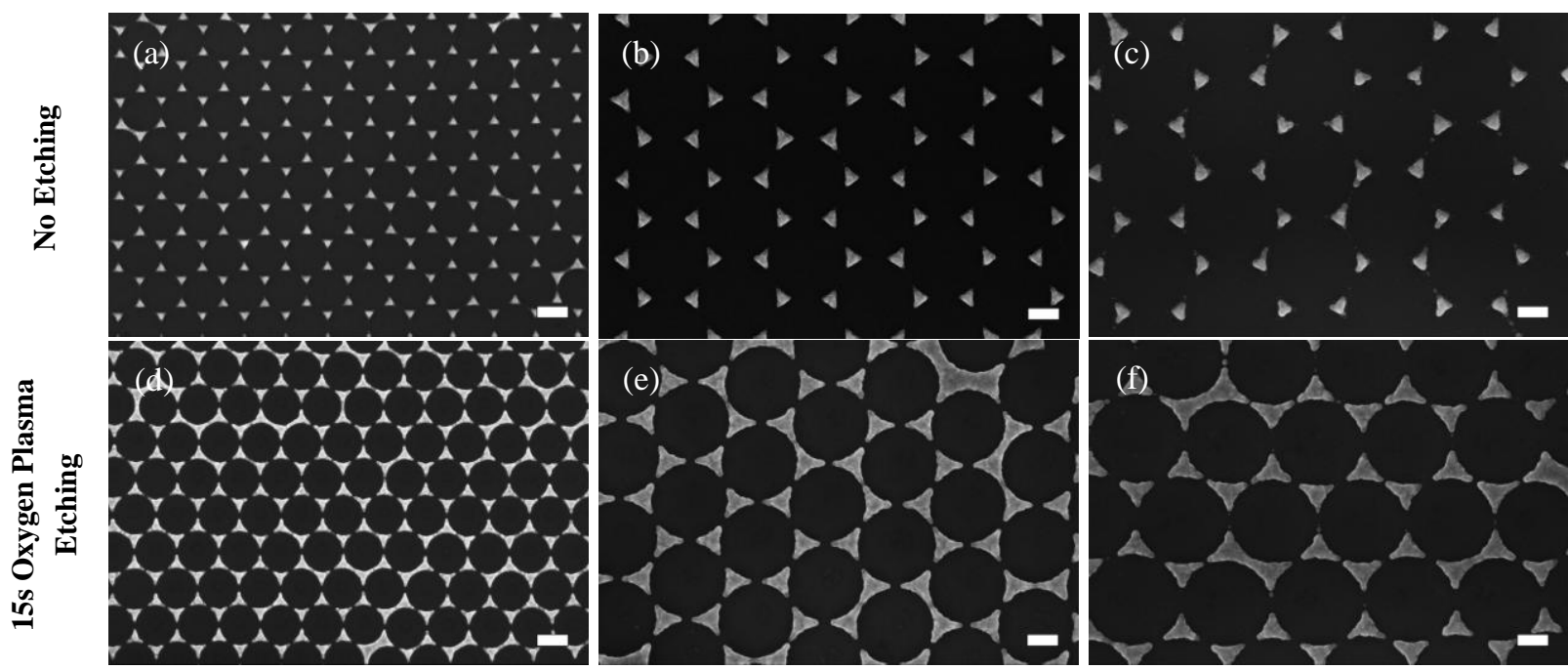

Figure 2 FEG-SEM images of Ag nanotriangular-like arrays: (a) $300 \mathrm{~nm}$ PS sphere template without oxygen plasma etching (PS300-0s), (b) $500 \mathrm{~nm}$ PS sphere template without oxygen plasma etching (PS500-0s), (c) $620 \mathrm{~nm}$ PS sphere template without oxygen plasma etching (PS620-0s), (d) $300 \mathrm{~nm}$ PS sphere template with 15 seconds oxygen plasma etching (PS300-15s), (e) $500 \mathrm{~nm}$ PS sphere template with oxygen plasma etching (PS500-15s), (f) $620 \mathrm{~nm}$ PS sphere template with oxygen plasma etching (PS620-15s) (scale bar: $200 \mathrm{~nm}$ ).

TABLE 1 Nanoparticle structural parameters corresponding to the Near- and Mid-Infrared Extinction ${ }^{\mathrm{a}}$

\begin{tabular}{ccccccc}
\hline Sample & PS300-0s & PS500-0s & P620-0s & PS300-15s & PS500-15s & PS620-15s \\
\hline $\mathbf{a}(\mathbf{n m})$ & $72 \pm 4$ & $118 \pm 11$ & $137 \pm 15$ & $110 \pm 8$ & $208 \pm 11$ & $227 \pm 23$ \\
$\mathbf{s}(\mathbf{n m})$ & $79 \pm 8$ & $160 \pm 16$ & $218 \pm 22$ & $40 \pm 4$ & $63 \pm 6$ & $80 \pm 8$ \\
$\mathbf{D}(\mathbf{n m})$ & 300 & 500 & 620 & 300 & 500 & 620 \\
$\boldsymbol{\lambda}_{\max }(\mathbf{n m})$ & 495 & 680 & 750 & 600 & 800 & 905 \\
$\boldsymbol{\lambda}_{\max }(\mathbf{n m})$ Calculated & 527 & 666 & 725 & 620 & 770 & 910 \\
\hline
\end{tabular}

${ }^{a}$ Measurements in Fig. 2 and 3 and FDTD calculations [(a) $300 \mathrm{~nm}$ PS sphere template without oxygen plasma etching (PS300-0s), (b) $500 \mathrm{~nm}$ PS sphere template without oxygen plasma etching (PS500-0s), (c) $620 \mathrm{~nm}$ PS sphere template without oxygen plasma etching (PS620-0s), (d) $300 \mathrm{~nm}$ PS sphere template with 15 seconds oxygen plasma etching (PS300-15s), (e) $500 \mathrm{~nm}$ PS sphere template with oxygen plasma etching (PS500-15s), (f) $620 \mathrm{~nm}$ PS sphere template with oxygen plasma etching (PS620-15s).

the original hcp arrangement of the PS sphere template. Fig. 2(a-c) shows Ag nanotriangular-like arrays after removal of the $300 \mathrm{~nm}, 500 \mathrm{~nm}$, and 620 $\mathrm{nm}$ PS sphere templates, respectively (Sample PS300-0s, PS500-0s, PS620-0s); while Fig. 2(d-f) shows enlarged Ag nanotriangular-like nanoparticles with smaller gaps by oxygen plasma etching of corresponding PS sphere templates before Ag evaporation (Sample PS300-15s, PS500-15s, PS620-15s). Their size distributions were obtained from at least 200 individual particles using ImageJ (http://rsbweb.nih.gov/ij/).

The in-plane width a, (tip to tip dimensions) and interparticle distances, of the $\mathrm{Ag}$ nanotriangular-like nanoparticles produced from the PS sphere templates, with and without oxygen plasma etching, are given in Table 1, along with the measured and calculated $\lambda_{\max }$. The height of the Ag nanotriangular-like arrays is $75 \mathrm{~nm}$, measured by a QCM of the Mantis evaporation system. The measured LSPR (Table 1), the signature optical property of noble metal nanoparticles, shows the systematic tunability of $\mathrm{Ag}$ nanotriangular-like arrays fabricated by colloidal lithography, in agreement with literature [33]. It is worth noting good agreement between modelling and experimental data, given the sensitivity of $\lambda_{\max }$ to nanoparticle size and the measured variation in sample size and separation. Here we observe that 

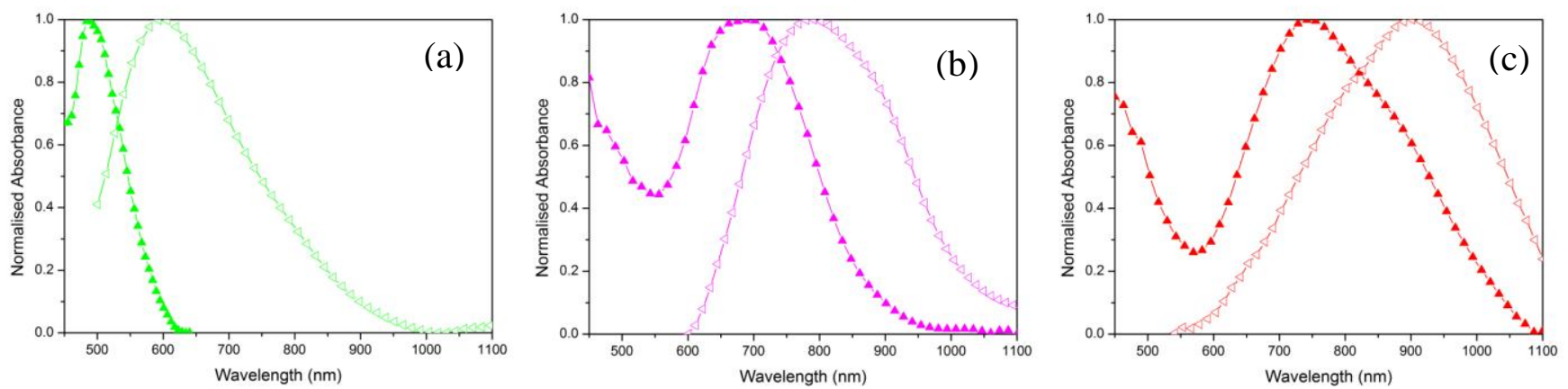

Figure 3 Plasmonic response of samples prepared using colloidal mask (with or without 15 seconds oxygen plasma etching): (a) PS300-0s and PS300-15s; (b) PS500-0s and PS500-15s; (c) PS620-0s and PS620-15s (solid triangle trace: without etching; hollow triangle trace: with 15 s etching).

the maximum difference between the calculated and experimental results is $\sim 6 \%$ (PS300-0s sample) and is between $0.0055 \%$ and $3.4 \%$ for the other samples. This approach allows us to design optimum nanoparticles arrays for future applications, and understand how imperfection affects the plasmonic response.

The extreme sensitivity of $\lambda_{\max }$ to nanoparticle size can be illustrated by comparing the LSPR spectra in Fig. 3. The nanoparticles that produce these spectra have identical shapes and approximately constant heights (shown in Fig. 2), but vary in the in-plane diameter, a, from $72 \mathrm{~nm}$ to $118 \mathrm{~nm}$, to $137 \mathrm{~nm}$ and result in a red shift of the $\lambda_{\max }$ from 495 to 680 to $750 \mathrm{~nm}$ respectively. This corresponds to an in-plane width sensitivity factor $\Delta \lambda_{\max } / \Delta \mathrm{a} \approx 4$, which is in good agreement with literature [34]. Previous work [35] on spherical particles has shown that the maximum MIFE would be expected at a slightly longer wavelength than the $\lambda_{\max }$. It has been shown that MIFE can be predicted by considering a dipole model for the fluorophore molecules [35], [36]. Whilst it is possible to predict MIFE for axis-symmetric spheres using FDTD, the lack of symmetry of triangular structures means that a large number of points around the particle would need to be considered. Each point would require a separate calculation and the total enhancement would then be the average. Nevertheless previous work on $\mathrm{Au}$ triangles [37] predicts that the optimum wavelength for MIFE to be slightly above $\lambda_{\max }$ for these triangular-like structures.
The primary consequences of LSPR excitation are selective photon absorption, scattering, and local electromagnetic field enhancement. The ability to manipulate and predict the LSPR of metal nanoparticle systems is desirable in several technological applications. The LSPR of metal nanoparticle systems, particularly those of $\mathrm{Ag}$ and $\mathrm{Au}$, is the source of the local electromagnetic field enhancement which is thought to be the dominant contribution to the large intensities observed in SERS. In this study, size-tunable Ag triangular-like nanoparticles fabricated by colloidal lithography provide outstanding control of nanoparticle size and interparticle spacing. The in-plane width and interparticle spacing can be independently tuned with nanometer precision by selection of the PS sphere diameter and oxygen plasma etching time. $\mathrm{Ag}$ triangular-like nanoparticles fabricated by colloidal lithography were then applied MIFE. Broad wavelength tunability was demonstrated as the $\lambda_{\max }$ shifts $2-5 \mathrm{~nm}$ per $1 \mathrm{~nm}$ variation in nanoparticles width. The effect of tunable LSPRs throughout the visible and near-infrared regions of the electromagnetic spectrum on MIFE was investigated by using Alexa Fluor dyes.

\subsection{Fluorescence Enhancement of Ag Nanostructures on nearby Fluorophores}

To establish the impact of the Ag triangular-like arrays prepared by colloidal lithography on nearby fluorophores, we chose three different streptavidin-Alexa Fluor conjugates (AF488-SA, AF680-SA, AF750-SA). As shown in Fig. 4(a-c), 

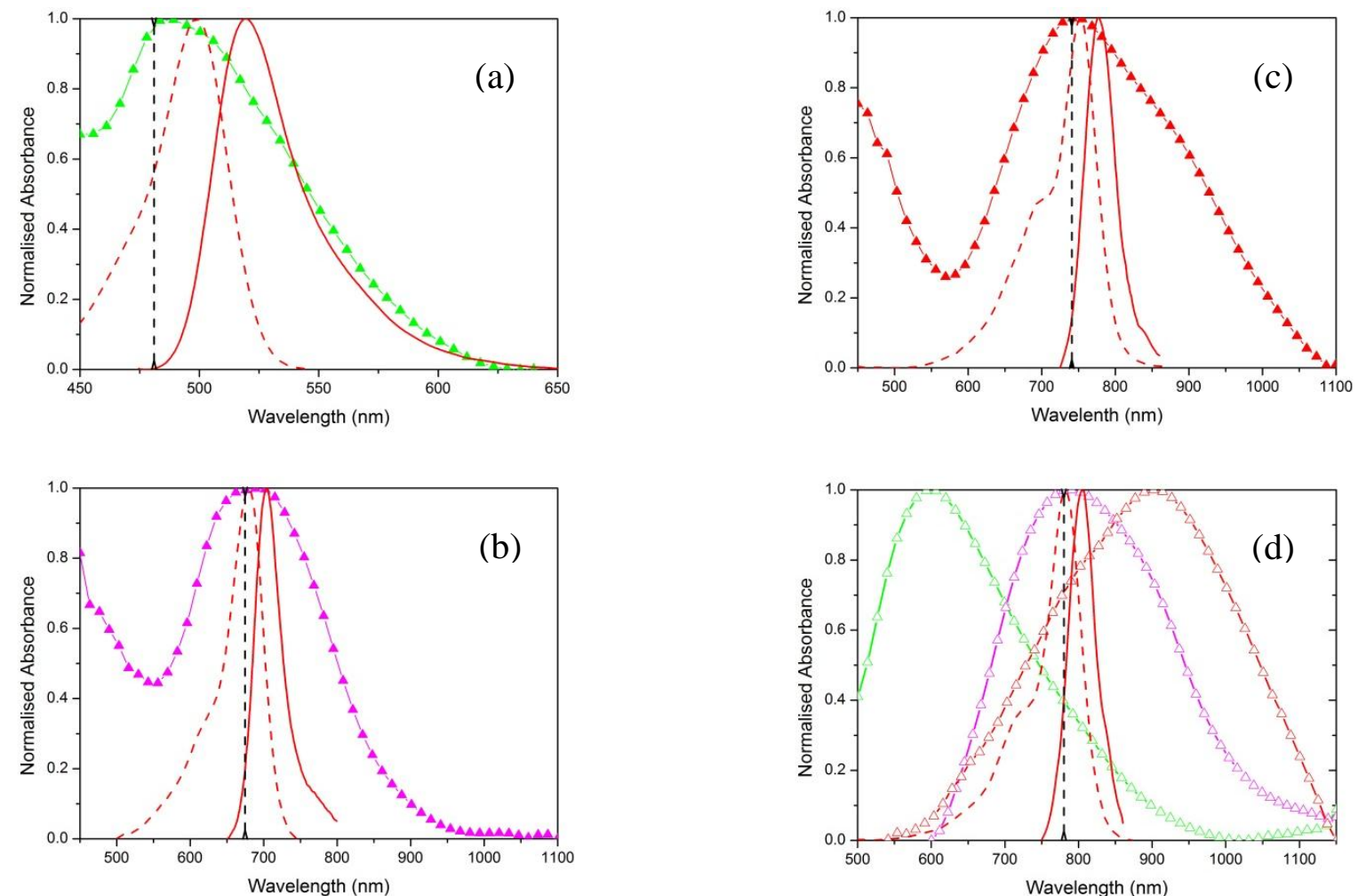

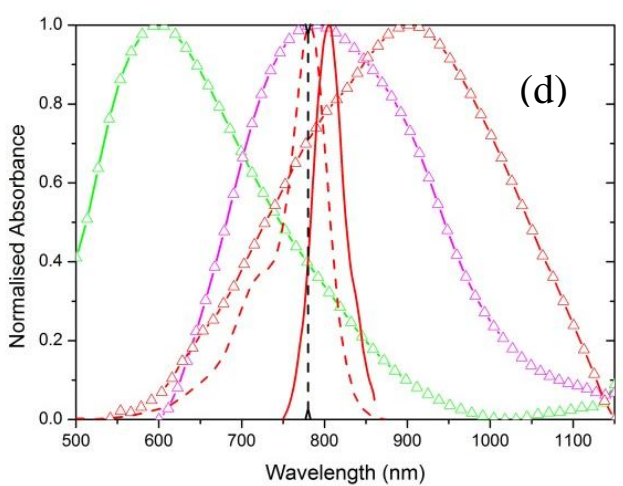

Figure 4 Normalized absorption and emission spectra (dotted and solid line traces, respectively) of Alexa Fluor dyes overlapping with corresponding LSPRs (black traces) of the set of the samples without oxygen plasma etching: (a) AF488-SA with LSPR of PS300 without etching; (b) AF680-SA with LSPR of PS500 without etching; (c) AF750-SA with LSPR of PS620 without etching. (d) AF790-SA with LSPRs of Ag triangular arrays from PS300, PS500, and PS620 templates after 15 second oxygen plasma etching, respectively. The excitation wavelength is indicated by the black dotted line.

AF488-SA, AF680-SA, and AF750-SA were chosen for maximum overlap of their absorption and emission spectra with the measured LSPR from the samples without oxygen plasma etching. It has been reported that enhanced fluorescence depends on the spectral overlap between the LSPR in metal nanostructures with spectral properties of the fluorophore [38]. Therefore, by maximizing the overlap of the absorption/emission spectra of dye with the LSPR, the maximum fluorescence enhancement is expected. For those samples with 15 seconds oxygen plasma etching, AF790-SA was selected to specifically study the fluorescence enhancement effect for NIR dye, as shown in Fig. 4(d). The selected fluorophores represent the spectral range from visible to near infrared that we wanted to study based on the measured LSPR response. Furthermore, they also represent a wide range of quantum efficiency (Qy) from high efficiency (Qy of AF488: 92\%) to medium efficiency
(Qy of AF680: 36\%) to low efficiency (Qy of AF750: 12\%; Qy of AF790: 4\%). In addition, the advantages of using Alexa Fluors, compared to conventional dyes, are their better photostabilities and less self-quenching when labelled with proteins [39].

It has been reported previously that bBSA binds to glass and gold/silver surfaces to form a complete monolayer [22] although with the different binding mechanism. The monolayer on glass surface is formed by non-covalent physisorbed binding while on metallic surface the bonding is believed to involve both electrostatic affinity and covalent binding. The streptavidin conjugated dyes were immobilized on substrates that were precoated with bBSA. The binding interaction between streptavidin and biotin is very strong $\left(\mathrm{K}_{\mathrm{A}} \approx 10^{15} \mathrm{M}^{-1}\right)$ and results in a stable monolayer of streptavidin-dye over the bBSA. The layer of bBSA serves also as a separation layer 

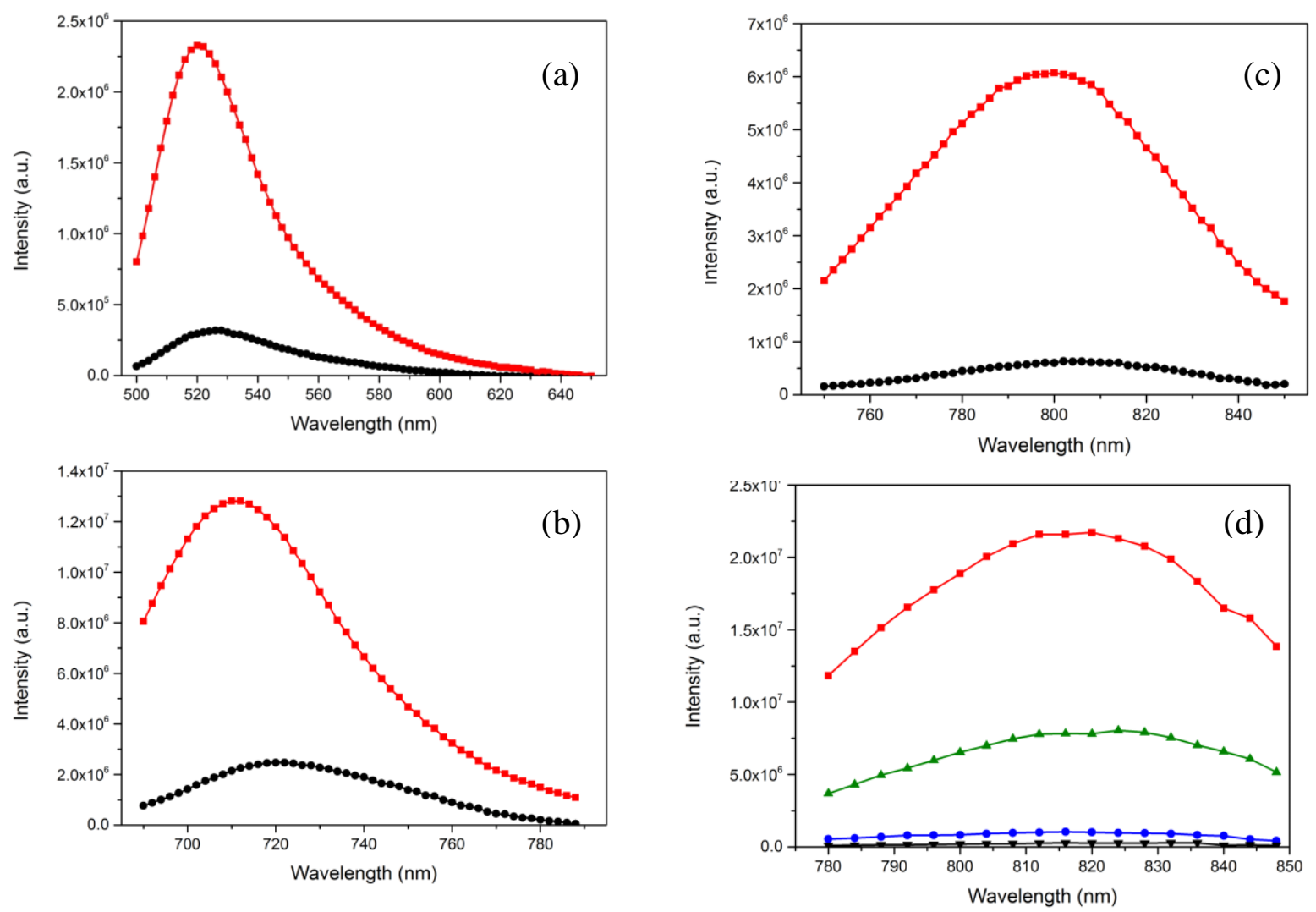

Figure 5 Fluorescence spectra of (a) Alexa Fluor 488 monolayer on sample PS300-0s (red) and on glass surface (black), (b) Alexa Fluor 680 monolayer on sample PS500-0s (red) and on glass surface (black), (c) Alexa Fluor 750 monolayer on sample PS620-0s (red) and on glass surface (black), (d) Alexa Fluor 790 monolayer on sample PS300-15s (blue), PS500-15s (red), and PS620-15s (green) as well as on glass as control (black), respectively.

Table 2 Average experimentally measured fluorescence enhancement factors of streptavidin conjugated Alexa Fluor dyes for the samples of PS300, PS500, and PS620 temples, with and without oxygen plasma etching

\begin{tabular}{cccc}
\hline Sample & $\begin{array}{c}\text { Fluorescence } \\
\text { Enhancement } \\
\mathrm{E}_{\mathrm{f}}\end{array}$ & Sample & $\begin{array}{c}\text { Fluorescence } \\
\text { Enhancement } \\
\mathrm{E}_{\mathrm{f}}\end{array}$ \\
\hline PS300-0s / & 7.8 & $\begin{array}{c}\text { PS300-15s / } \\
\text { AF790-SA }\end{array}$ & 5.5 \\
AF488-SA & & PS500-15s/ & 83.0 \\
PS500-0s / & 5.7 & AF790-SA & \\
AF680-SA & & PS620-15s/ & 33.8 \\
PS620-0s / & 10.0 & AF790-SA & \\
AF750-SA & & &
\end{tabular}

${ }^{\mathrm{a}}$ Data were corrected for variations in streptavidin-dye conjugation surface coverage for different samples. between the fluorophores and the Ag surface. Since
bBSA has an ellipsoid shape with two axes of 4 and $8 \mathrm{~nm}$, the true thickness of the absorbed bBSA molecules is in between these two extreme values. Additionally, streptavidin, which conjugates with dyes and forms a monolayer of about $4 \mathrm{~nm}$ thickness, acts as an additional separation layer between the Ag surface and fluorophore. Such a combined spacing of about $10 \mathrm{~nm}$ provides the optimum condition for MIFE by minimizing nonradiative decay channels, which can lead to fluorescence quenching at a small $(<4 \mathrm{~nm})$ distances [23][30].

Figure 5, shows the fluorescence measurement of the four fluorophores bound to the corresponding Ag nanostructures and fluorescence enhancement observed. The enhancement factors are summarized in Table 2, after normalising for surface area differences. The graph shows the 
well-established fluorescence spectra of Alexa Fluor dyes bound to proteins, where the samples were excited at $480 \mathrm{~nm}, 670 \mathrm{~nm}, 740 \mathrm{~nm}$, and $780 \mathrm{~nm}$ for conjugates AF488-SA, AF680-SA, AF750-SA, and AF790-SA, respectively. The highest fluorescence enhancement observed was from AF790-SA on the sample of PS500-15s, which was almost 2 orders of magnitude greater than that of the AF790-SA monolayer deposited on glass. The enhancement factors are 7.8, 5.7, 10.0 for AF488-SA, AF680-SA, AF750-SA for PS300, PS500, and PS620 templates without etching, respectively, as shown in Table 2.

We estimated the excess surface area due to $\mathrm{Ag}$ nanostructures to be less than $20 \%$ compared with the planar glass substrate. Therefore, the observed large fluorescence enhancement cannot be related simply to differences in the surface concentration of bound fluorophore-streptavidin conjugates and must be due to surface enhancement phenomena. In view of sensing applications, the increase in the sensing active area is desirable for improved sensitivity and sensing dynamic range.

We did not observe significantly higher enhancement factors from low quantum yield dyes in our samples, which is in agreement with previously reported results [41]. It is clear that there is some ambiguity in the results for the un-etched samples, as the fluorescence enhancement for the lower QY AF680-SA (with the PS500-0s template) is lower than the PS300-0s/AF488-SA sample. Since we excite close to the $\lambda_{\max }$ for both templates we had expected that the fluorescent enhancement would be higher for the AF680-SA dye. We do not currently fully understand this result and so we are undertaking a FDTD analysis of emission and excitation enhancement from fluorophore molecules around these Ag triangular like structures. Whilst still at an early stage we have observed that fluorescent enhancement drops off very rapidly just below the main plasmon resonant peak and rises again at shorter wavelengths due to higher order LSPR modes. It has also been reported that for spherical nanoparticles the fluorescent enhancement for Ag drops quicker as the wavelength increases above $\lambda_{\max }$ compared to Au nanoparticles. Hence, for these triangular like particles with large separation distances it is likely that there is a very narrow spectral window where optimum MIFE is achievable. The electromagnetic coupling between closely spaced nanoparticles would appear to increase this bandwidth. Table 2 also shows the enhancement factors for AF790-SA are 5.5, 83.0, and 33.8, for PS300, PS500, and PS620 templates with 15 seconds oxygen plasma etching, respectively. The data were averaged over three samples for each case, in addition to 3 different locations from single sample.

We observed much higher fluorescence enhancement for those samples with 15 seconds oxygen plasma etching, partially due to the low quantum efficiency of AF790 and much smaller gap size between nanoparticles after etching. It is well-known that local electromagnetic field enhancement is highly dependent on the gap size, with a smaller gap size causing plasmon coupling and dramatically enhancing the field close to the metal particle [30]. Such a field enhancement is one of the two factors contributing to fluorescence enhancement as discussed previously. It should be emphasized that for these results fluorescence enhancement is measured over relatively large areas of $1 \mathrm{~mm}$ by $3 \mathrm{~mm}$ compared to literature results which are often local measurements on the scale of micrometers [23]. Such an observed fluorescence enhancement of Ag nanostructures on fluorophores is attributed to the interplay of two principal factors: an increase of a local electromagnetic field near Ag nanostructures, leading to an increased excitation rate of the fluorophores, and an increase of the radiative decay rate of fluorophores close to metal nanostructures, reflected both in the fluorescence lifetime and quantum yield [40]. The local EM field enhancement produces a higher excitation rate, but it does not change the lifetime of the fluorophore; this effect is referred to as excitation enhancement. The second effect known as emission enhancement increases the quantum yield. The increased quantum yield and thus the emission enhancement were for AF790-SA monolayer on the samples with 15 seconds oxygen plasma etching.

Figure 6 shows the simulated electric field enhancement at the excitation wavelength of the 
AF790-SA for PS300, PS500 and PS620 templates with $15 \mathrm{~s}$ oxygen plasma etching. It can be seen that for the PS500 template with etching the field enhancement in the proximity of the metal particle is 10 or greater. The other two cases show less field enhancement and for the PS300 case the significant enhancement is only seen in the space between the particles. The enhanced electric near-field around the metal excites the fluorophore which then emits at the emission wavelength, $\lambda_{\mathrm{em}}$. At $\lambda_{\mathrm{em}}$ the

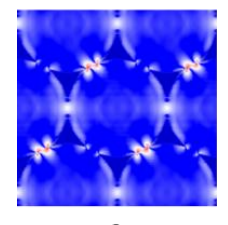

a

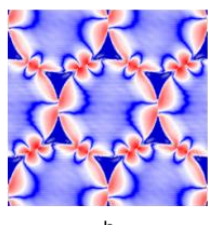

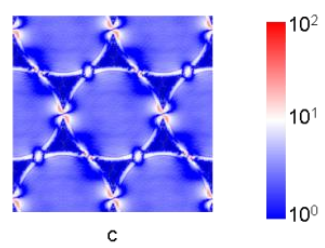

Figure 6 Plots of relative electric field enhancement around the metal nanoparticles at $780 \mathrm{~nm}$ excitation wavelength, for three templates (a) PS300 (b) PS500 (c) PS620 with 15s etching.

oscillating dipole and couples energy to the metal nanoparticle. It should be noted that the plasmonic peak is only an indicator of the wavelength of maximum possible MIFE. This is because the local electromagnetic environment must be modified by the fluorophore in order for significant electromagnetic coupling to occur between the molecule and metal. Further, the dipolar coupling between the metal and the molecule is not present in either the far-field calculations, or measurements of scattering and absorption. An important aspect in MIFE is the electric field enhancement around the metal which causes excitation enhancement. The electric field plots also indicate points from where emission enhancement can be expected. This is because if there is little, or no, Stokes shift it would be expected that strongest electromagnetic coupling would occur from points where large electric fields are seen around the metal during excitation. This is based on the fact that we must see reciprocity in the coupling between the metal and fluorophore, if the quantum yield of the dye is one and there is no Stoke's shift.

\subsection{Lifetime Measurement for AF790-SA Monolayers}

To prove that the observed fluorescence enhancement factors are mediated by the LSPR effect in Ag nanostructures, we performed fluorescence lifetime measurements for the samples with 15 s oxygen plasma etching, to provide insights into fluorescence decay rates and to semi-quantitatively deconvolute the total fluorescence enhancement by experimental measurement of emission enhancement. Fig. 7 shows the fluorescence lifetime spectra of AF790-SA monolayer on PS300, PS500, and PS620 template with etching, as well as on a glass surface as a control. The average lifetime of AF790-SA monolayer on glass was measured to be 550 ps. The lifetimes of AF790-SA monolayer on PS300, PS500, and PS620 templates with oxygen plasma etching were reduced significantly to 238 ps, 113 ps, and 177 ps, respectively.

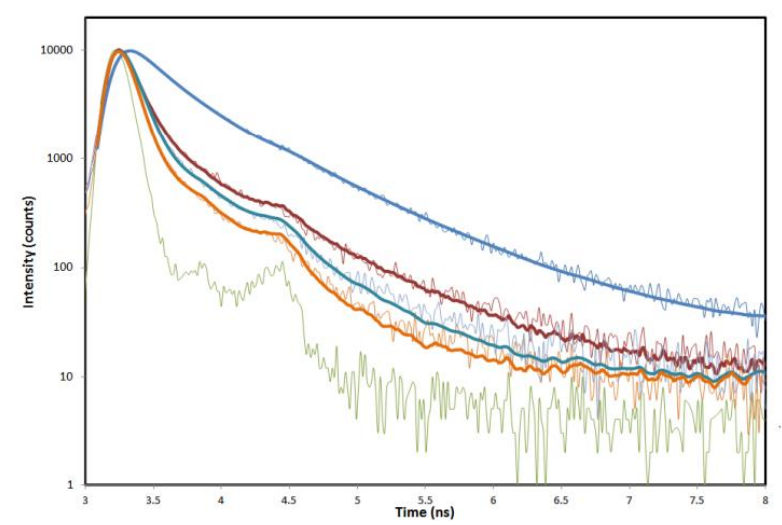

Figure 7 Time domain fluorescence decay of AF790-SA monolayer on (a) glass ( $\tau \sim 550 \mathrm{ps}$ ), (b) on PS300 template with 15 sec oxygen plasma etching ( $\tau \sim 238$ ps), (c) on PS500 template with $15 \mathrm{sec}$ oxygen plasma etching ( $\tau \sim 113 \mathrm{ps}$ ), (d) on PS620 template with $15 \mathrm{sec}$ oxygen plasma etching $(\tau \sim 177$ ps).

The fluorescence lifetime data can be evaluated in terms of a single exponential (SE) model or a multiexponential (ME) model. A SE model is appropriate for samples consisting of a single fluorophore in a homogeneous environment, while a ME model describes the fractional contribution of decay time for each component present in a sample mixture [42]. In our case, the fluorescence decay curves could be satisfactorily fitted using a model with two decay times $t_{1}$ and $t_{2}$, from which an average value is calculated using the weighting $a_{1}$ and $a_{2}$ for each of the decay components: 


$$
I(t)=a_{1} e^{-t / t_{1}}+a_{2} e^{-t / t_{2}}
$$

The results of fitting to a ME decay analysis are represented in Table 3. The $x^{2} \mathrm{R}$ values shown in the last column of Table 3 are the goodness of fit parameter, obtained by fitting calculated values to experimentally obtained parameters by a nonlinear least-squares deconvulation method. The $x^{2} \mathrm{R}$ represented here are $<10 \%$ of the random deviations in the data [42].

The lifetime results shown above were analysed within the framework of a widely accepted semi-empirical model [41]. The observed emission of the fluorophore in the absence of any quenching interactions is described in terms of quantum yield $\left(\mathrm{Q}_{0}\right)$ and lifetime $\left(\tau_{0}\right)$. The quantum yield is the fraction of the excited fluorophores which relax by radiative decay $\left(\Gamma_{0}\right)$ relative to the total relaxation rate $\left(\Gamma_{0}+k_{n r}\right)$, given by:

$$
Q_{0}=\frac{\Gamma_{0}}{\Gamma_{0}+k_{n r}}
$$

The observed lifetime is simply the inverse of the total decay rate of the excited state:

$$
\tau_{0}=\frac{1}{\Gamma_{0}+k_{n r}}
$$

where $\mathrm{kn}_{\mathrm{n}}$ is the non-radiative decay rate. The subscript ' 0 ' indicates the fluorophore is isolated and not in the presence of a metal nanoparticle.

Table 3 Multiexponential analysis of intensity decay of AF790-SA monolayer on glass surface and metallic surface, showing weighting fraction $\left(\mathrm{a}_{1}, \mathrm{a}_{2}\right)$, observed lifetime $\left(\tau_{1}, \tau_{2}\right.$, $\mathrm{ps})$, intensity weighted lifetime $(\langle\tau\rangle, \mathrm{ps})$, and goodness of fit parameter $\left(x_{\mathrm{R}}^{2}\right)$.

\begin{tabular}{ccccccc}
\hline Sample & $\mathrm{a}_{1}$ & $\mathrm{a}_{2}$ & $\tau_{1}$ & $\tau_{2}$ & $\tau$ & $x_{\mathrm{R}}^{2}$ \\
\hline $\begin{array}{c}\text { AF790-SA on } \\
\text { glass }\end{array}$ & $46.3 \%$ & $53.7 \%$ & 264 & 722 & 510 & 1.40 \\
$\begin{array}{c}\text { AF790-SA on } \\
\text { PS300-15s }\end{array}$ & $93.3 \%$ & $6.7 \%$ & 84 & 560 & 238 & 1.75 \\
$\begin{array}{c}\text { AF790-SA on } \\
\text { PS500-15s }\end{array}$ & $96.8 \%$ & $3.2 \%$ & 51 & 374 & 113 & 1.70 \\
$\begin{array}{c}\text { AF790-SA on } \\
\text { PS620-15s }\end{array}$ & $94.5 \%$ & $5.5 \%$ & 77 & 464 & 177 & 1.72 \\
\hline
\end{tabular}

When a fluorophore is in the proximity of a metal nanoparticle, the enhanced near field of the nanoparticle increases the amount of energy absorbed by the fluorophore. In addition, electromagnetic coupling occurs between the fluorophore and the nanoparticle plasmon, causing an increase in the radiative decay rate of the molecule at the emission wavelength. The proximity of fluorophore to metals results in an increase in the total radiative decay rate by addition of a new rate $\Gamma_{\mathrm{m}}$. The modified quantum yield $\left(\mathrm{Q}_{\mathrm{m}}\right)$ and lifetime $\left(\tau_{\mathrm{m}}\right)$ are then give by:

$$
\begin{gathered}
\mathrm{Q}_{\mathrm{m}}=\frac{\Gamma_{\mathrm{m}}+\Gamma_{0}}{\Gamma_{\mathrm{m}}+\Gamma_{0}+\Gamma_{\mathrm{m}, \mathrm{abs}}+\mathrm{k}_{\mathrm{nr}}} \\
\tau_{\mathrm{m}}=\frac{1}{\Gamma_{\mathrm{m}}+\Gamma_{0}+\Gamma_{\mathrm{m}, \mathrm{abs}}+\mathrm{k}_{\mathrm{nr}}}
\end{gathered}
$$

where $\Gamma_{\mathrm{m}}$, abs is the additional nonradiative decay rate. The effectiveness of $\Gamma_{\mathrm{m}}$ abs falls off rapidly with separation distance, thus the modified quantum yield tends back to the original quantum yield at far enough separation. For short fluorophore-metal distances, $<4 \mathrm{~nm}$, the non-radiative energy transfer rate depends on the inverse cube of the molecule-surface separation [43]. In other words, the fluorophore is quenched by the metal nanoparticle until complete quenching occurs at very small separation distance. However, in our case, a streptavidin-biotin conjugate provides a spacer layer of $12 \mathrm{~nm}$ between the fluorophore and the Ag nanoparticle surface. For this significantly larger metal-fluorophore distance, a significant increase in non-radiative decay rate due to metal nanoparticles is not anticipated. Therefore, we assume that non-radiative decay rate is essentially the same for Alex Fluor dyes on glass surface and on metallic surface, which means $\Gamma_{\mathrm{m}, \mathrm{abs}}$ can be neglected.

By using the measured fluorescence lifetime of AF790-SA on clean glass surface the quantum yield of F790-SA in free-space conditions of 0.1, and Eq. 2-3 and 4-5, we were able to obtain the value of $\Gamma_{\mathrm{m}} / \Gamma$ on $\mathrm{Ag}$ nanostructures relative to the rate on unmodified glass and hence the emission enhancement $\left(E_{\mathrm{em}}\right)$, defined as $E_{\mathrm{em}}=Q_{\mathrm{m}} / Q_{0}$, for each 
sample. These are listed in Table 4. The excitation enhancement $\left(E_{\mathrm{ex}}\right)$ for each sample is calculated by dividing the (total) fluorescence enhancement factor $\left(E_{\mathrm{f}}\right)$ by the emission enhancement $\left(Q_{\mathrm{m}} / Q_{0}\right)$, given by $E_{\mathrm{ex}}=E_{\mathrm{f}} / E_{\mathrm{em}}$.The values are listed in Table 5.

The fluorescence lifetime study presented here provides insight into fluorescence decay rates. The lifetimes on metallic surfaces (shown in Table 4) are all shorter than the AF790-SA lifetime on the glass

Table 4 Lifetime measurements for each sample and the calculated values of lifetime, radiative rate, and quantum yield ratios on metallic surfaces versus clean glass surface. $\left(\mathrm{Q}_{0}\right.$ $=10 \%$ )

\begin{tabular}{cccccc}
\hline Sample & $\begin{array}{c}\tau_{\mathrm{m}} \\
(\mathrm{ps})\end{array}$ & $\tau / \tau_{\mathrm{m}}$ & $\left(\Gamma_{\mathrm{m}}+\Gamma\right) / \Gamma$ & $\mathrm{Q}_{\mathrm{m}}$ & $\begin{array}{c}\mathrm{E}_{\mathrm{em}} \\
=\mathrm{Q}_{\mathrm{m}} / \mathrm{Q}_{0}\end{array}$ \\
\hline PS300-15s & 238 & 2.14 & 2.4 & $21.0 \%$ & 2.1 \\
PS500-15s & 113 & 4.51 & 26.1 & $72.3 \%$ & 7.2 \\
PS620-15s & 177 & 2.88 & 9.8 & $52 \%$ & 5.2 \\
\hline
\end{tabular}

Table 5: Values of the Excitation Enhancement and Emission Enhancement for Each Sample

\begin{tabular}{cccc}
\hline Sample & $\mathrm{E}_{\mathrm{f}}$ & $\mathrm{E}_{\mathrm{em}}$ & $\mathrm{E}_{\mathrm{ex}}$ \\
\hline PS300-15s & 5.5 & 2.1 & 2.6 \\
PS500-15s & 83.0 & 7.2 & 11.5 \\
PS620-15s & 33.8 & 5.2 & 6.5 \\
\hline
\end{tabular}

surface. The radiative decay rates are significantly increased and likewise the quantum yields of the fluorophore. The shortest observed lifetime for the AF790-SA monolayer on PS500-15s is 113 ps, which is well within the range of experimental capabilities and accuracy, since the fluorescence lifetime system response is of the order of 30 ps. The high quantum yield of $72.3 \%$ for sample PS500-15s demonstrated that plasmonic enhancement can be used to create NIR dyes with similar brightness as visible dyes. Also, we observed that both the fluorescence enhancement factor and lifetime follow exactly opposite trends with the shortest lifetime (for PS500-15s) correlating with the highest enhancement factor, which indicates that the effect of metal-nanostructure-mediated fluorescence enhancement takes place in the present nanostructures. As shown in Table 5, it is possible to semi-quantitatively separate excitation enhancement from emission enhancement, the two main mechanisms for the observed fluorescence enhancement. The values are in good agreement with our electric field mapping, which indicates sample PS500-15s has the highest field enhancement at $780 \mathrm{~nm}$. We use the term "semi-quantitatively" because the precise relative contribution of each process responsible for fluorescence enhancement is difficult to determine, since we also need to consider the coupling efficiency of the fluorescence emission to the far field. Nonetheless, the values in Table 5 give us an indication of significant impact of LSPR on fluorescence enhancement. The overlapping degree of LSPR to excitation/emission of nearby dye is the key to have maximum fluorescence enhancement.

\section{Conclusions}

In conclusion it has been demonstrated that florescence intensity enhancement is possible in the NIR using Ag nanostructures produced by colloidal lithography. In our experiments an enhancement of 83 was obtained for NIR dye Alex Fluor 790. It is shown that interparticle separation, particle resonance, and the fluorescent dye properties of quantum yield and emission/excitation wavelengths are all important factors for fluorescence enhancement.

This work clearly demonstrates that MIFE at NIR wavelengths is possible using Ag nanoparticle patterned surfaces manufactured from colloidal lithography. The colloidal lithography technique allows fabrication of large area arrays of $\mathrm{Ag}$ triangular nanoparticles with controlled size, shape, and interparticle separation in a relatively low-cost manner, with tunable optical features. These arrays are robust and reproducible and are an excellent candidate for practical sensing applications which require high sensitivity and reproducibility.

\section{Acknowledgements}

This work was supported by UK EPSRC Programme 
Grant (EP/G060940/1). F. Xie is supported by the Imperial College JRF scheme. J. Pang acknowledges financial support by King Abdullah University of Science and Technology. We thank Dr. L. Danos for PicoQuant lifetime measurement.

Electronic Supplementary Material: Supplementary material (Further Details of the FDTD Modeling) is available in the online version of this article at http://dx.doi.org/10.1007/s12274-***_******

(automatically inserted by the publisher).

\section{References}

[1] Lakowicz, J. R. Plasmonics 2006, 1, 5-33.

[2] Lakowicz, J. R. Probe Design and Chemical Sensing; Lakowicz, J. R., Ed.; Plenum Press: New York, 1994; Vol. 4.

[3] Luo, S.; Zhang, E.; Su, Y.; Cheng, T.; Shi, C. Biomaterials 2011, 32, 7127-7138.

[4] Licha, K.; Riefke, B.; Ntziachristos, V.; Becker, A.; Chance, B.; Semmier, W. Photochem. Photobiol. 2000, 72, 392-398.

[5] Kronick, M. N. J. Immunol. Methods 1986, 92, 1-13.

[6] Near-Infrared Dyes for High Technology Applications; Daehne, S.; Resch-Genger, U.; Wolfbeis, O. S., Eds.; Kluwer Academic Publishers: Dordrecht, The Netherlands, 1998.

[7] Walker, N. J. Science 2002, 296, 557-559.

[8] Xie, F.; Baker, M. S.; Goldys, E. M. J. Phys. Chem. B 2006, 110, 23085-23091.

[9] Purcell, E. M. Phys. Rev. 1946, 69, 674.

[10] Dulkeith, E.; Ringler, M.; Klar, T. A.; Feldman, J.; Munoz Javier, A.; Parak, W. J. Nano Lett. 2005, 5, 585-589.

[11] Dulkeith, E.; Morteani, A. C.; Niedereichholz, T.; Klar, T. A.; Feldman, J.; Levi, S. A.; van Voggel, F. C. J. M.; Reinhoudt, D. N.; Moller, M.; Gittins, D. I. Phys. ReV. Lett. 2002, 89, 203002.

[12] Cannone, F.; Chirico, G.; Bizzari, A. R.; Cannistraro, S. J. Phys.Chem. B 2006, 110, 16491-16498.

[13] Pan, S. L.; Wang, Z. J.; Rothberg, L. J. J. Phys. Chem. B 2006,110, 17383-17387.

[14] Kulakovich, O.; Strekal, N.; Yaroschevich, A.; Maskevich, S.; Gaponenko, S.; Nabiev, L.; Woggon, U.; Artemyev, M. Nano Lett. 2002, 2, 1449-1452.

[15] Shimizu, K. T.; Woo, W. K.; Fisher, B. R.; Eisler, H. J.; Bawendi, M. G. Phys. Rev. Lett. 2002, 89, 117401.

[16] Lakowicz, J. R. Anal. Biochem. 2005, 337, 171-194.

[17] Xie, F.; Centeno, A.; Ryan, M. P.; Riley, D. J.; Alford, N. M. J. Mater. Chem. B 2013, 1, 536-543.

[18] Bardhan, R.; Grady, N. K.; Cole, J. R.; Joshi, A.; Halas, N. J. ACS nano 2009, 3, 744-752.

[19] Tabakman, S. M.; Lau, L.; Robinson, J. T.; Price, J.; Sherlock, S. P.; Wang, H.; Zhang, B.; Chen, Z.; Tangsombatvisit, S.; Jarrell, J. A.; Utz, P. J.; Dai, H. J.
Nature Communications 2011, 2, 466.

[20] Wang, D.; Mohwald, H. Adv. Mater. 2004, 16, 244-247.

[21] Zhou, L.; Ding, F.; Chen, H.; Ding, W.; Zhang, W.; Chou, S. Y. Anal. Chem. 2012, 84, 4489-4495.

[22] Malicka, J.; Gryczynski, I.; Gryczynski, Z.; Lakowicz, J. Anal. Biochem. 2003, 315, $57-66$.

[23] Bharadwaj, P.; Novotny, L. Opt. Express 2007, 15, 14266-14274.

[24] Anderson, J. P.; Griffiths, M.; Boveia, V. R. Plasmonics 2006, 1, 103-110.

[25] Anderson, J. P.; Griffiths, M.; Williams, J. G.; Grone, D. L.; Lamb, D. L.; Boveia V. R. In Proceedins of SPIE 6641, Plasmonics: Metallic Nanostructures and Their Optical Properties V, San Diego, CA, 2007, 664108.

[26] Geddes, C. D.; Cao, H.; Gryczynski, I.; Gryczynski, Z.; Fang, J.; Lakowicz, J. R. J. Phys. Chem. A 2003, 107, 3443-3449.

[27] Danos, L.; Markvart, T. Chem. Phys. Lett. 2010, 490, 194-199.

[28] Taflove, A.; Hagness, S. C. Computational electrodynamics: The finite difference time-domain method, Artech House, 2005, 3rd edn.

[29] Rakic, A. D.; Djurisic, A. B.; Elavar, J. M.; Majewski, M. L. Appl. Opt. 1998, 37, 5271-5283.

[30] Centeno, A.; Xie F.; Alford, N. M. J. Opt. Soc. Am. B 2011, 28, 325-330.

[31] Oskooi, A. F.; Roundy, D.; Ibanescu, M.; Bermel, P.; Joannopoulos, J. D.; Johnson, S. G. Comput. Phys. Commun. 2010, 181, 687-702.

[32] Jain, P. K.; El-Say, M. A. Nano Lett. 2007, 7, 2854-2858.

[33] Kinkhabwala, A.; Yu, Z.; Fan, S.; Avlasevich, Y.; Mullen, K.; Moerner, W. E. Nat. Photonics 2009, 3, 654-657.

[34] Jensen, T. R.; Malinsky, M. D.; Haynes, C. L.; Van Duyne, R. P. J. Phys. Chem. B 2000, 104, 10549-10556.

[35] Centeno, A.; Xie F.; Alford, N. M. IET Nanobiotechnology 2013, IET digital library identifier: NBT-2012-0016.R1.

[36] Vukovic, S.; Corni, S.; Mennucci, B. J. Phys. Chem. C 2009, $113,121-133$.

[37] Centeno, A.; Xie, F.; Alford, N. M. A Computational Study of the Coupled Emissions between Flurophores and Gold Triangular Prism Bow Tie, Progress In Electromagnetics Research Symposium Proceedings, KL, MALAYSIA, 2012, March 27, 30, 765-767.

[38] Tomsia, K.; Xie F.; Goldys, E.; J. Phys. Chem. C 2010, 114, $1562-1569$.

[39] Panchuk-Voloshina, N.; Haugland, R. P.; Biship-Stewart, J.; Bhalgat, M. K.; Millard, P. J.; Mao, F.; Leung, W. Y.; Haugland, R. P. J. J. Histochem. Cytochem. 1999, 47, 1179-1188.

[40] Sokolov, K.; Chumanov, G.; Cotton, T. M. Anal. Chem. 1998, 70, 3898-3905.

[41] Draga, A. I.; Geddes, C. D. Appl. Phys. Lett. 2012, 100, 093115.

[42] Lakowicz, J. R. Time-Domain Lifetime Measurements. In Principles of Fluorescence Spectroscopy, 2nd ed.; Kluwer Academic/Plenum: New York, 1999; pp95-184.

[43] Axelrod, D.; Hellen, E.; Fulbright, R. Topics in fluorescence spectroscopy, 2002, 289-343. 


\title{
Electronic Supplementary Material
}

\section{Further Details of the FDTD Modeling}

\author{
Fang Xie ${ }^{1}(\varangle)$, Jing S. Pang, ${ }^{1}$ Anthony Centeno, ${ }^{2}$ Mary P. Ryan, ${ }^{1}$ D. Jason Riley ${ }^{1}$, and Neil M. Alford ${ }^{1}$ \\ ${ }^{1}$ Department of Materials and London Centre for Nanotechnology, Imperial College London, London, SW7 2AZ, UK \\ ${ }^{2}$ Malaysia Japan International Institute of Technology, University Technology Malaysia International Campus, Jalan Semarak, 54100, \\ Kuala Lumpur, Malaysia \\ Received: day month year / Revised: day month year / Accepted: day month year (automatically inserted by the publisher) \\ (c) Tsinghua University Press and Springer-Verlag Berlin Heidelberg 2011 \\ Supporting information to DOI $10.1007 / \mathrm{s} 12274-* * * *_{-} * * * *_{-} *$ (automatically inserted by the publisher)
}

In all the Finite Difference Time Domain (FDTD) calculations the array is considered to be in free-space and we calculate the scattering coefficients and the absorption. The transmission and reflection coefficients are calculated by considering the power flow through computational surfaces above and below the nanoparticle array, as depicted in Figure 1 and previously described in reference [1]. The transmission and reflection is normalized to the incident power through the top computational surface. The normalized absorption is then found by subtracting the total scattering from one, where the total scattering is the sum of the normalized transmission and reflection coefficients. Although the scattering from the particles will occur over all angles the fact that the array is periodic and the computational surfaces extend over the entire computational domain means that all the scattered field, either reflected or transmitted, will pass through either the top or bottom computational surface. A number of simulations were executed for each nanoparticle size and configuration to find appropriate distances of the computational surfaces from the silver triangular like nanoparticles.

The top and bottom boundaries of the computational domain are terminated using perfectly matched layers (PML's) which prevent any non-physical reflections. The other four FDTD edges of the computational domain are periodic boundaries which creates the infinite two-dimensional array of particles. The incident electric field upon the nanoparticle array is circularly polarized and is normally incident. 


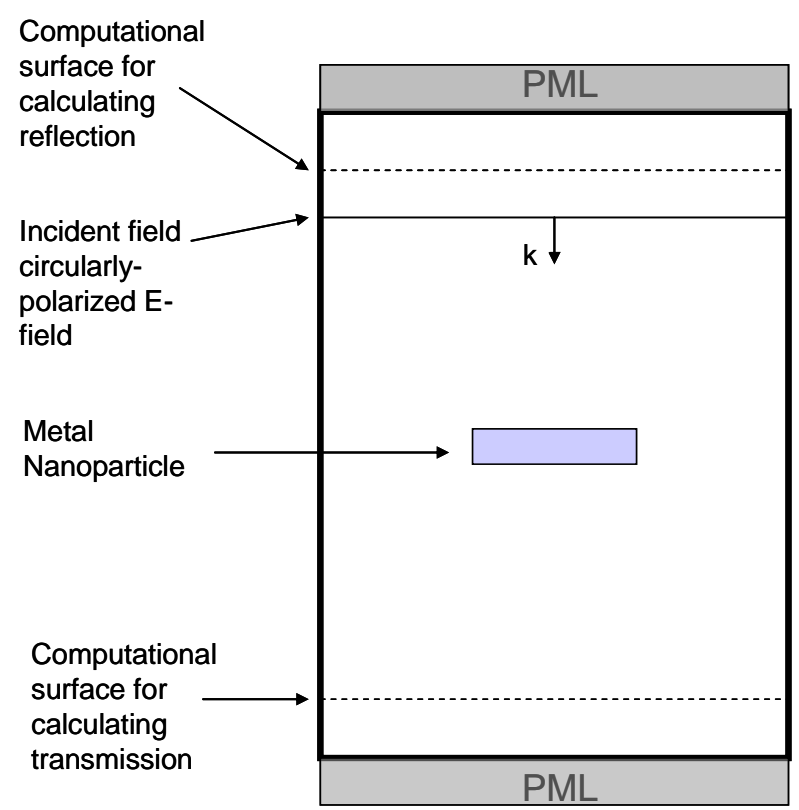

Fig. S-1 Depiction of FDTD computational domain (in cross-section) showing the position of computational surfaces used to find transmission and reflection.

The metal nanoparticles were modelled using the combined Drude-Lorentz model for metal permittivity [2]:

$$
\varepsilon(\omega)=\varepsilon_{\infty}+\sum_{i=1}^{n} \frac{\alpha_{i} \omega_{p}^{2}}{\omega_{o i}^{2}-\omega^{2}-j \omega \tau_{i}}
$$

where $\omega p$ is the plasma frequency, $\alpha$ is the oscillators' strength, wo is the resonant frequency of each oscillator, $\mathrm{j}$ is the imaginary unit and $\tau$ is the damping frequency of each oscillator. In our calculations we have used one Drude and five Lorentz terms in the summation. The parameters used were obtained from Rakic et-al [2] and are shown in Table 1.

Table S-1. Parameters used in FDTD calculations for silver. (Parameters obtained from reference [2])

\begin{tabular}{|l|l|l|l|l|l|l|}
\hline \multicolumn{2}{|l|}{ Silver $\omega \mathrm{p}=9.01 \mathrm{eV}$} \\
\hline $\mathrm{i}$ & 1 & 2 & 3 & 4 & 5 & 6 \\
\hline$\alpha$ & 0.845 & 0.065 & 0.124 & 0.011 & 0.840 & 5.646 \\
\hline$\tau(\mathrm{eV})$ & 0.048 & 3.886 & 0.452 & 0.065 & 0.916 & 2.419 \\
\hline$\omega 0 \quad(\mathrm{eV})$ & 0.000 & 0.816 & 4.481 & 8.185 & 9.083 & 20.29 \\
\hline
\end{tabular}

\section{References}

1. A. Centeno, F. Xie and N. Alford, J. Opt. Soc. Am. B, 28, 2011, 325-330.

2. A.D.Rakic, A.B.Djurisic, J.M.Elavar and M.L.Majewski, Appl. Opt. 37, 1998, 5271-5283. 